\title{
Association of Thyroid Function Test Abnormalities and Thyroid Autoimmunity With Preterm Birth A Systematic Review and Meta-analysis
}

The Consortium on Thyroid and Pregnancy-Study Group on Preterm Birth

IMPORTANCE Maternal hypothyroidism and hyperthyroidism are risk factors for preterm birth. Milder thyroid function test abnormalities and thyroid autoimmunity are more prevalent, but it remains controversial if these are associated with preterm birth.

OBJECTIVE To study if maternal thyroid function test abnormalities and thyroid autoimmunity are risk factors for preterm birth.

DATA SOURCES AND STUDY SELECTION Studies were identified through a search of the Ovid MEDLINE, EMBASE, Web of Science, the Cochrane Central Register of Controlled Trials, and Google Scholar databases from inception to March 18, 2018, and by publishing open invitations in relevant journals. Data sets from published and unpublished prospective cohort studies with data on thyroid function tests (thyrotropin [often referred to as thyroid-stimulating hormone or TSH] and free thyroxine [ $\left.\mathrm{FT}_{4}\right]$ concentrations) or thyroid peroxidase (TPO) antibody measurements and gestational age at birth were screened for eligibility by 2 independent reviewers. Studies in which participants received treatment based on abnormal thyroid function tests were excluded.

DATA EXTRACTION AND SYNTHESIS The primary authors provided individual participant data that were analyzed using mixed-effects models.

MAIN OUTCOMES AND MEASURES The primary outcome was preterm birth ( $<37$ weeks' gestational age).

RESULTS From 2526 published reports, 35 cohorts were invited to participate. After the addition of 5 unpublished data sets, a total of 19 cohorts were included. The study population included 47045 pregnant women (mean age, 29 years; median gestational age at blood sampling, 12.9 weeks), of whom 1234 (3.1\%) had subclinical hypothyroidism (increased thyrotropin concentration with normal $\mathrm{FT}_{4}$ concentration), 904 (2.2\%) had isolated hypothyroxinemia (decreased $\mathrm{FT}_{4}$ concentration with normal thyrotropin concentration), and 3043 (7.5\%) were TPO antibody positive; 2357 (5.0\%) had a preterm birth. The risk of preterm birth was higher for women with subclinical hypothyroidism than euthyroid women (6.1\% vs 5.0\%, respectively; absolute risk difference, $1.4 \%$ [95\% $\mathrm{Cl}, 0 \%-3.2 \%]$; odds ratio [OR], 1.29 [95\% Cl, 1.01-1.64]). Among women with isolated hypothyroxinemia, the risk of preterm birth was $7.1 \%$ vs $5.0 \%$ in euthyroid women (absolute risk difference, $2.3 \%[95 \% \mathrm{Cl}$, 0.6\%-4.5\%]; OR, 1.46 [95\% Cl, 1.12-1.90]). In continuous analyses, each 1-SD higher maternal thyrotropin concentration was associated with a higher risk of preterm birth (absolute risk difference, $0.2 \%$ [95\% Cl, 0\%-0.4\%] per 1SD; OR, 1.04 [95\% Cl, 1.00-1.09] per 1SD). Thyroid peroxidase antibody-positive women had a higher risk of preterm birth vs TPO antibody-negative women ( $6.6 \%$ vs $4.9 \%$, respectively; absolute risk difference, $1.6 \%$ [95\% Cl, 0.7\%-2.8\%]; OR, 1.33 [95\% Cl, 1.15-1.56]).

CONCLUSIONS AND RELEVANCE Among pregnant women without overt thyroid disease, subclinical hypothyroidism, isolated hypothyroxinemia, and TPO antibody positivity were significantly associated with higher risk of preterm birth. These results provide insights toward optimizing clinical decision-making strategies that should consider the potential harms and benefits of screening programs and levothyroxine treatment during pregnancy. 
$\mathrm{P}$ reterm birth complicates $5 \%$ to $15 \%$ of births worldwide. It is the most important direct cause of morbidity and mortality in children younger than 5 years, ${ }^{1}$ and is an important risk factor for psychiatric, metabolic, cardiovascular, and renal disease later in life. ${ }^{2,3}$ However, in the majority of cases, no known risk factors can be identified. 4,5

Thyroid hormone regulates metabolism, growth, and development in most tissues of the human body, including various physiological processes related to preterm birth, such as placental development and function, fetal growth, and expression of neuropeptides involved in the onset of labor. ${ }^{6-9}$ Overt hypothyroidism and hyperthyroidism during pregnancy are well-known risk factors for preterm birth and occur in approximately $0.5 \%$ and $0.05 \%$ of pregnancies, respectively. ${ }^{10,11}$ During pregnancy, thyroid function test abnormalities (subclinical hypothyroidism and isolated hypothyroxinemia) and thyroid autoimmunity are much more frequent than overt thyroid disease.$^{11}$ However, it remains to be determined whether such thyroid function test abnormalities are risk factors for preterm birth.

Estimates for the association of subclinical hypothyroidism or isolated hypothyroxinemia with preterm birth in previous observational studies range from odds ratios (ORs) of 0.57 to 3.3. ${ }^{12-15}$ The interpretation of these studies is limited by 2 main factors. First, the studies have used different upper limits for thyrotropin (often referred to as thyroid-stimulating hormone or TSH; ranging from $>2.5$ to $>6.0 \mathrm{mIU} / \mathrm{L}$ ). Second, most are single-center studies and lack statistical power. Furthermore, only a few studies have investigated whether isolated hypothyroxinemia could be a risk factor for adverse pregnancy outcomes.

This study assessed whether thyroid function test abnormalities and thyroid peroxidase (TPO) antibody positivity were associated with preterm birth.

\section{Methods}

The Consortium on Thyroid and Pregnancy is a collaboration of prospective birth cohorts that aims to study the association of maternal thyroid function and autoimmunity with adverse pregnancy and child outcomes. For the current study, we followed Preferred Reporting Items for Systematic Reviews and Meta-Analyses guidelines for Individual Patient Data and preregistered the study protocol(CRD42016043494), which appears in Supplement 1 along with an outline of protocol deviations.

To identify studies for inclusion, we conducted a systematic literature search for articles on the association of thyroid function or autoimmunity with preterm birth published from database inception to March 18, 2018, without language restrictions, using the Ovid MEDLINE, EMBASE, Web of Science, Cochrane Central Register of Controlled Trials, and Google Scholar databases (eMethods in Supplement 2). In addition, unpublished data were identified via open invitations sent to relevant journals, ${ }^{16,17}$ international conferences, social media, and personal contacts. The search was repeated on June 19, 2019, to identify studies

\section{Key Points}

Question Are thyroid function test abnormalities or thyroid autoimmunity a risk factor for preterm birth?

Findings In this individual participant data meta-analysis of 19 prospective cohort studies including 47045 women, subclinical hypothyroidism (odds ratio [OR], 1.29), isolated hypothyroxinemia (OR, 1.46), and thyroid peroxidase antibody positivity $(O R, 1.33)$ were each significantly associated with a higher risk of preterm birth.

Meaning These findings provide evidence that subclinical hypothyroidism, isolated hypothyroxinemia, and thyroid peroxidase antibody positivity in pregnant women are risk factors for preterm birth.

published after March 18, 2018, that would have been eligible for inclusion.

We included prospective cohort studies that included participants consecutively without selection criteria related to health status (such as comorbidities or thyroid disease) and had data on thyrotropin concentration, free thyroxine $\left(\mathrm{FT}_{4}\right)$ concentration, or TPO antibody measurements and data on gestational age at birth. We excluded studies in which participants received treatment based on abnormal thyroid function tests (predominantly hospital-based cohorts) or studies that only included women with overt thyroid disease.

Possible studies for inclusion were independently assessed for suitability by 2 of the authors (T.I.M.K. and P.N.T.) and any disagreement was resolved by discussion with a third author (R.P.P.). Investigators from each eligible study were invited to join the consortium. Study quality and risk of bias were assessed using the Newcastle-Ottawa scale.

Local institutional review board approval and participant informed consent was obtained for all separate cohorts; no additional approvals were obtained for the current study. After obtaining individual participant data, all participants with data on thyrotropin concentration, $\mathrm{FT}_{4}$ concentration, or TPO antibody concentration, and gestational age at birth were included. We excluded participants who had a miscarriage or perinatal death, preexisting thyroid disease, thyroid-interfering medication use, in vitro fertilization, or multiple pregnancies.

\section{Primary and Secondary Outcomes}

The primary outcome was preterm birth defined as a gestational age at birth of less than 37 weeks. Gestational age was assessed using either ultrasound measurements or time after the last menstrual period. Secondary outcomes were very preterm birth ( $<32$ weeks' gestational age) and the gestational age at birth.

\section{Exposures}

Exposure variables included thyroid function test abnormalities, continuous thyroid function test measurements (thyrotropin and $\mathrm{FT}_{4}$ concentrations), TPO antibody positivity, and thyroglobulin antibody positivity. Thyroid function test abnormalities were defined according to cohort-specific 
2.5th and 97.5th population percentiles for thyrotropin and $\mathrm{FT}_{4}$ concentrations after exclusion of TPO antibody-positive women. Subclinical hypothyroidism was defined as a thyrotropin concentration above the 97.5th percentile and a $\mathrm{FT}_{4}$ concentration within the normal range (ie, 2.5th-97.5th percentile). Overt hyperthyroidism was defined as a thyrotropin concentration below the 2.5 th percentile and a $\mathrm{FT}_{4}$ concentration above the 97.5th percentile. Subclinical hyperthyroidism was defined as a thyrotropin concentration below the 2.5th percentile and a $\mathrm{FT}_{4}$ concentration within the normal range. Isolated hypothyroxinemia was defined as a $\mathrm{FT}_{4}$ concentration below the 2.5 th percentile and a thyrotropin concentration within the normal range. Thyroid peroxidase antibody positivity and thyroglobulin antibody positivity were defined in a cohort-specific manner according to cutoffs recommended by the manufacturer. For analyses with continuous thyrotropin and $\mathrm{FT}_{4}$ concentrations as exposure variables, the concentrations for all cohorts were logtransformed and then standardized to population-specific standard deviation scores after removal of outliers $( \pm 4 \mathrm{SD}$ from the mean) to enable comparisons among different cohorts and assays.

\section{Statistical Analyses}

\section{Primary Analyses}

We studied the association of thyroid function test abnormalities (the reference group was women with normal thyroid), thyrotropin and $\mathrm{FT}_{4}$ concentrations continuously, and TPO and thyroglobulin antibody positivity with preterm birth and very preterm birth using generalized linear mixed models with a random intercept for each cohort. For the same exposures, the association with gestational age at birth was studied using linear mixed models with a random intercept for each cohort.

The primary analyses were repeated with a 2-step approach by using random-effect models according to the DerSimonian and Laird method to pool estimates and the Firth bias reduction method in case of near or complete separation in smaller cohorts. Heterogeneity across studies was assessed using the $I^{2}$ statistic. To evaluate potential publication bias, funnel plots and Egger tests were used. All analyses were adjusted for maternal age, body mass index (BMI), ethnicity, smoking, parity, gestational age at blood sampling, and fetal sex. We used multilevel multiple imputation ${ }^{18}$ for missing data on covariates. Five imputed data sets were created and pooled for analyses using Rubin rules. ${ }^{19}$

\section{Secondary and Sensitivity Analyses}

We performed a prespecified sensitivity analysis for which analyses on thyroid function or thyroid function test abnormalities were additionally adjusted for TPO antibody positivity. A post hoc sensitivity analysis assessed whether the association of TPO antibody positivity with preterm birth and very preterm birth differed for each increase in continuous thyrotropin concentration by adding a product interaction term into the models and stratification of analyses according to thyrotropin cutoffs recommended by the guidelines of the American Thyroid Association. ${ }^{20}$ To assess TPO antibody positivity, a post hoc sensitivity analysis explored different cutoffs based on a recent study showing a dose-dependent effect on thyroid function (alternative cutoffs: 94th population percentile, higher absolute concentrations of $>100$ and $>500 \mathrm{mIU} / \mathrm{L}$, and higher relative concentrations of $2 \times$ and $5 \times$ the assay cutoff). ${ }^{21}$ In addition, a prespecified analysis explored whether the association of thyrotropin concentration, $\mathrm{FT}_{4}$ concentration, or TPO antibody positivity differed according to differences in gestational age at the time of blood sampling, BMI, or parity.

A 2-sided threshold for statistical significance of $<.05$ was used. Because no adjustment was made for multiple testing, findings from the secondary analyses and sensitivity analyses should be interpreted as exploratory. All statistical analyses were performed using R version 3.4.4 (R Project for Statistical Computing).

\section{Results}

From 2526 published reports, 133 remained eligible for inclusion based on screening of the title and abstract (Figure 1). After reading the full text, a total of 35 cohorts were invited to participate. Five unpublished data sets were added via personal contacts and responses from open invitations. Four studies were published after the index date of our systematic search that otherwise would have been eligible for inclusion. A total of 19 cohorts from Europe, the United States, Chile, Australia, Pakistan, and Japan responded to the invitation and were able to participate.

After exclusions, the final study population comprised 47045 participants (Figure 1), of whom 1234 (3.1\%) had subclinical hypothyroidism (increased thyrotropin concentration with normal $\mathrm{FT}_{4}$ concentration), 904 (2.2\%) had isolated hypothyroxinemia (decreased $\mathrm{FT}_{4}$ concentration with normal thyrotropin concentration), and 3043 (7.5\%) were TPO antibody positive. Preterm birth and very preterm birth occurred in 2357 (5.0\%) and 349 (0.7\%) pregnancies, respectively (Table). Cohort-specific population characteristics, details on data collection, cohort-specific number of participants with available thyroid function and gestational age at birth, data quality assessment by the Newcastle-Ottawa scale (indicating high-quality data for all cohorts), and cohort-specific percentile cutoffs for thyroid function test abnormalities (thyrotropin and $\mathrm{FT}_{4}$ concentrations) appear in eTables 1-4 in Supplement 2.

Data on specific covariates were missing for up to $33 \%$ of the women and from as many as 3 cohorts (total percentage of missing data for maternal age: $0.3 \%$ [0 cohorts]; gestational age at the time of blood sampling: $0.4 \%$ [0 cohorts]; parity: $5.8 \%$ [ 1 cohort]; smoking status: $7.2 \%$ [ 1 cohort]; sex of the child: $21.4 \%$ [ 2 cohorts]; BMI: $32.9 \%$ [3 cohorts]). The women that were not included due to missing data on gestational age at birth had similar mean standardized thyrotropin concentrations in SDs vs those who were included (-0.03 vs 0, respectively; $P=.28)$, but different mean standardized $\mathrm{FT}_{4}$ concentrations in SDs (0.06 vs $0 ; P=.05)$ and different proportions with TPO antibody positivity $(13.0 \%$ vs $7.5 \% ; P<.001)$ (eTable 5 in Supplement 2). 


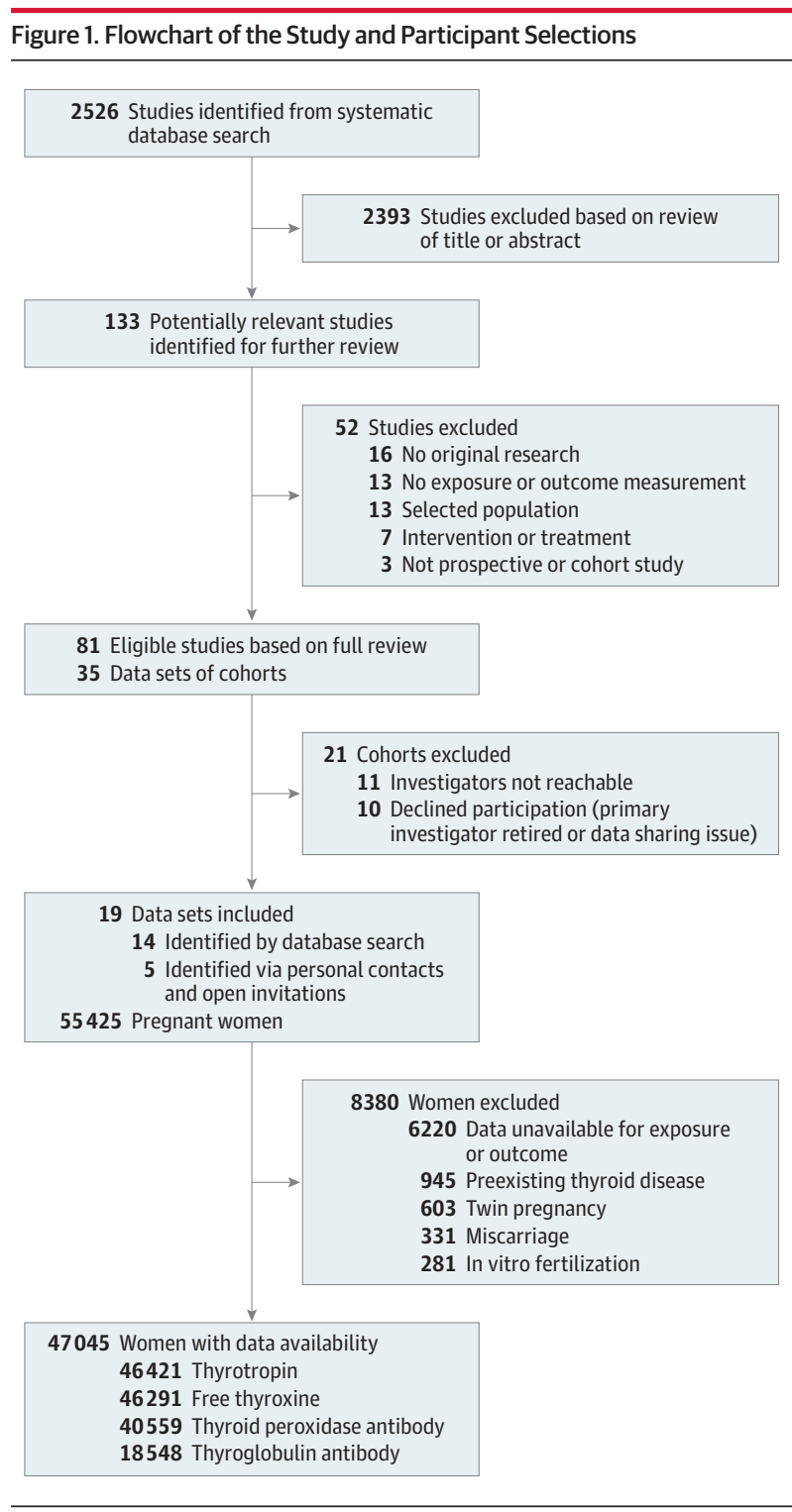

Preterm and Very Preterm Birth Thyroid Function Test Abnormalities

Women with subclinical hypothyroidism had a higher risk of preterm birth vs euthyroid women (6.1\% vs $5.0 \%$, respectively; absolute risk difference, $1.4 \%$ [95\% CI, 0\% to $3.2 \%$ ]; OR, 1.29 [95\% CI, 1.01 to 1.64$])$, but not of very preterm birth $(0.7 \%$ vs $0.8 \%$; absolute risk difference, $0 \%$ [ $95 \% \mathrm{CI},-0.3 \%$ to $0.8 \%$ ]; OR, 1.03 [95\% CI, 0.52 to 1.20]; Figure 2). Women with isolated hypothyroxinemia had a higher risk of preterm birth vs euthyroid women (7.1\% vs 5.0\%, respectively; absolute risk difference, $2.3 \%$ [ $95 \%$ CI, 0.6\% to $4.5 \%$ ]; OR, 1.46 [95\% CI, 1.12 to 1.90$])$ and a higher risk of very preterm birth (1.9\% vs $0.8 \%$; absolute risk difference, $1.2 \%$ [95\% CI, 0.4\% to $2.5 \%$ ]; OR, 2.57 [95\% CI, 1.55 to 4.27]). Among women with overt hyperthyroidism, there was no statistically significant difference in the rate of preterm birth vs euthyroid women (4.0\% vs $5.0 \%$, respectively; absolute risk difference, $-1.2 \%$ [95\% CI, $-2.8 \%$ to 1.7\%]; OR, 0.76 [95\% CI, 0.43 to 1.34]; Figure 2).

\begin{tabular}{|c|c|c|}
\hline & $\begin{array}{l}\text { No. of Participants/ } \\
\text { Total No. }(\%)^{\mathrm{a}}\end{array}$ & $\begin{array}{l}\text { Median } \\
\text { (95\% Range) }\end{array}$ \\
\hline \multicolumn{3}{|l|}{ Maternal Demographics } \\
\hline Age, y & 46893 & $29.0(5.2)^{\mathrm{b}}$ \\
\hline $\begin{array}{l}\text { Gestational age } \\
\text { at blood sampling, wk }\end{array}$ & 46876 & $12.9(7.0-39.7)$ \\
\hline Body mass index ${ }^{c}$ & 31728 & $23.9(4.4)^{\mathrm{b}}$ \\
\hline \multicolumn{3}{|l|}{ Parity } \\
\hline 0 & 24 180/44 $336(54.5)$ & \\
\hline 1 & $13217 / 44336(29.8)$ & \\
\hline 2 & $4435 / 44336(10.0)$ & \\
\hline$\geq 3$ & $2504 / 44336(5.6)$ & \\
\hline \multicolumn{3}{|l|}{ Smoking status } \\
\hline $\begin{array}{l}\text { Nonsmoker or } \\
\text { past smoker }\end{array}$ & $38733 / 43662(88.7)$ & \\
\hline Current smoker & 4929/43 $662(11.3)$ & \\
\hline \multicolumn{3}{|l|}{ Education level $^{\mathrm{d}}$} \\
\hline Low & $9677 / 31574$ (30.6) & \\
\hline Middle & $10647 / 31574(33.7)$ & \\
\hline High & $11250 / 31574(35.6)$ & \\
\hline \multicolumn{3}{|l|}{ Maternal Concentrations } \\
\hline Thyrotropin, mIU/L & 46421 & $1.33(0.13-4.52)$ \\
\hline Free thyroxine, pmol/L & 46291 & $13.0(7.2-21.9)$ \\
\hline $\begin{array}{l}\text { Thyroid peroxidase } \\
\text { antibody positivity }\end{array}$ & $3043 / 40559(7.5)$ & \\
\hline $\begin{array}{l}\text { Thyroglobulin } \\
\text { antibody positivity }\end{array}$ & $1080 / 18548(5.8)$ & \\
\hline \multicolumn{3}{|l|}{ Pregnancy Characteristics } \\
\hline $\begin{array}{l}\text { Gestational age } \\
\text { at birth, wk }\end{array}$ & 47045 & $39.9(35.3-42.0)$ \\
\hline \multicolumn{3}{|l|}{ Sex } \\
\hline Male & $18936 / 37355$ (50.7) & \\
\hline Female & $18419 / 37355$ (49.3) & \\
\hline $\begin{array}{l}\text { Late preterm birth } \\
\text { (gestational age }<37 \mathrm{wk})^{\mathrm{f}}\end{array}$ & $2357 / 47045(5.0)$ & \\
\hline $\begin{array}{l}\text { Early preterm birth } \\
\text { (gestational age <32 wk) }\end{array}$ & $349 / 47045(0.7)$ & \\
\hline
\end{tabular}

SI conversion factor: To convert free thyroxine to $\mathrm{ng} / \mathrm{dL}$, divide by 12.871 .

${ }^{a}$ The total No. indicates the No. of participants with available data on the thyroid function tests. Cohort-specific descriptive characteristics appear in eTable 1 in Supplement 2.

${ }^{\mathrm{b}}$ Expressed as mean (SD).

'Calculated as weight in kilograms divided by height in meters squared.

${ }^{\mathrm{d}}$ Defined as estimated years of education beyond primary school (low: 0-4 years; middle: $4-8$ years; high: >8 years).

e Often referred to as thyroid-stimulating hormone or TSH.

f Includes very preterm birth (<32 weeks' gestational age).

\section{Continuous Analyses of Thyroid Function}

Continuous analyses showed that each 1-SD higher maternal thyrotropin concentration was significantly associated with a $4 \%$ higher relative risk of preterm birth (absolute risk difference, $0.2 \%$ [95\% CI, 0\% to 0.4\%] per 1 SD; OR, 1.04 [95\% CI, 1.00 to 1.09 ] per $1 \mathrm{SD}$ ), but not very preterm birth (absolute risk difference, $0 \%$ [ $95 \%$ CI, $-0.05 \%$ to $0.10 \%$ ] per 1 SD; OR, 1.04 [ $95 \%$ CI, 0.93 to 1.16] per 1 SD; Figure 3 and eTable 6 in Supplement 2). Each 1-SD higher maternal $\mathrm{FT}_{4}$ concentration was not associated with preterm birth, but was significantly associated with a $12 \%$ lower risk of very preterm birth (absolute risk 


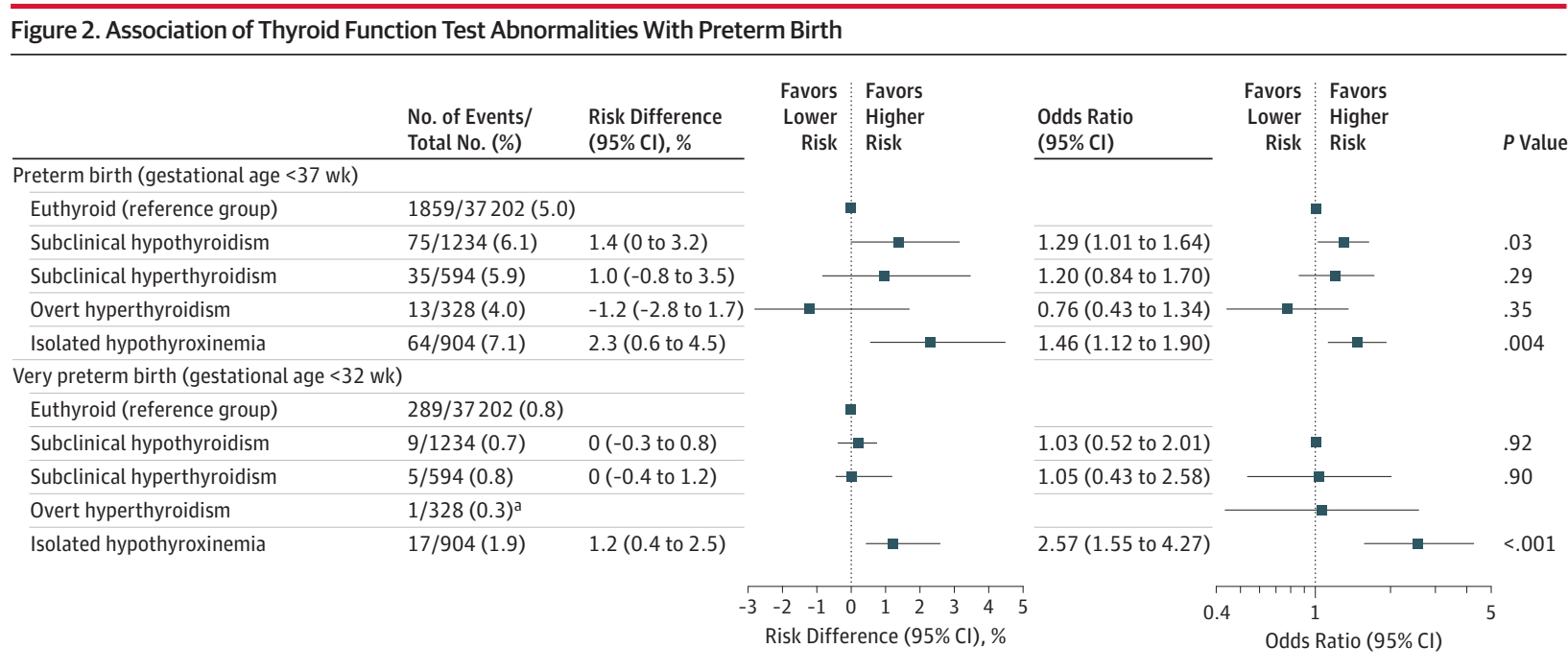

All analyses were adjusted for maternal age, body mass index, ethnicity, smoking, parity, gestational age at blood sampling, and fetal sex. Euthyroid was defined as the 2.5th-97.5th cohort-specific percentile for thyrotropin (often referred to as thyroid-stimulating hormone or TSH) and free thyroxine $\left(\mathrm{FT}_{4}\right)$ concentrations; subclinical hypothyroidism, increased thyrotropin concentration with a normal $\mathrm{FT}_{4}$ concentration; subclinical hyperthyroidism, decreased thyrotropin concentration with a normal $\mathrm{FT}_{4}$ concentration; overt hyperthyroidism, decreased thyrotropin concentration with an increased
$\mathrm{FT}_{4}$ concentration; and isolated hypothyroxinemia, a normal thyrotropin concentration with a decreased $\mathrm{FT}_{4}$ concentration. These clinical entities were calculated for cohorts that had thyrotropin concentration, $\mathrm{FT}_{4}$ concentration, and thyroid peroxidase antibody data available. Absolute differences and corresponding $95 \% \mathrm{Cls}$ were back-calculated from the results of multivariable models and adjusted for baseline risk imprecision.

a There were too few samples to conduct a reliable analysis. difference, $-0.10 \%$ [95\% CI, $-0.20 \%$ to $-0.01 \%$ ] per $1 \mathrm{SD}$; OR, 0.88 per 1 SD [ $95 \%$ CI, 0.79 to 0.95]; Figure 3 and eTable 6 in Supplement 2).

\section{TPO Antibody and Thyroglobulin Antibody Positivity}

Thyroid peroxidase antibody-positive women had a higher risk of preterm birth vs TPO antibody-negative women (6.6\% vs $4.9 \%$, respectively; absolute risk difference, $1.6 \%$ [95\% CI, $0.7 \%$ to $2.8 \%$ ]; OR, 1.33 [95\% CI, 1.15 to 1.56 ]; Figure 4$)$ and very preterm birth (1.7\% vs $0.7 \%$; absolute risk difference, $1.0 \%$ [95\% CI, 0.6\% to 1.7\%]; OR, 2.45 [95\% CI, 1.81 to 3.32]; Figure 4). There was no significant association of thyroglobulin antibody positivity with preterm birth vs those with thyroglobulin antibody negativity (4.3\% vs $4.4 \%$, respectively; absolute risk difference, $-0.5 \%$ [95\% CI, $-1.6 \%$ to $0.9 \%$ ]; OR, 0.88 [95\% CI, 0.64 to 1.20 ]; eTable 6 in Supplement 2).

\section{Gestational Age at Birth}

The results relating to the gestational age at birth were similar to those for preterm and very preterm birth (eTable 7 in Supplement 2).

\section{Sensitivity Analyses}

In the prespecified sensitivity analysis, the results for thyrotropin concentration, $\mathrm{FT}_{4}$ concentration, and TPO antibody positivity remained similar when all 3 exposures were added to the same model, but the association of subclinical hypothyroidism with preterm birth was no longer statistically significant after additional adjustment for TPO antibody positivity (eTable 8 in Supplement 2).
In the post hoc sensitivity analysis on the association of TPO antibody positivity with preterm and very preterm birth, the $P$ value for interaction for risk of preterm birth with higher thyrotropin concentrations among TPO antibodypositive women was .08; for very preterm birth, $P<.001$ for interaction; and for gestational age at birth continuously, $P=.006$ for interaction. Subsequent stratified analyses showed that TPO antibody-positive women and a thyrotropin concentration above $4.0 \mathrm{mIU} / \mathrm{L}$ had an excess risk of preterm birth vs TPO antibody-negative women (7.1\% vs $4.9 \%$, respectively; absolute risk difference, $2.7 \%$ [95\% CI, 0.2\%6.3\%]; OR, 1.55 [95\% CI, 1.05-2.27]). However, stratified analyses could not be performed for very preterm birth (Figure 4).

In a post hoc sensitivity analysis, alternative TPO antibody concentration cutoffs were significantly associated with a higher risk of preterm birth, but the results were similar to those identified for manufacturer-based cutoffs (eTable 9 in Supplement 2). In the prespecified analysis on the association of thyroid function with preterm birth, TPO antibody positivity or thyroglobulin antibody positivity did not differ significantly according to the gestational age at the time of blood sampling, BMI, or parity $(P>.07$ for interaction for all analyses without meaningful differences after stratification; eTables 10 and 11 in Supplement 2).

The primary analyses were similar using a 2-step approach; however, some cohort-specific analyses with very few cases exhibited inflated ORs (eFigures 1 and 2 in Supplement 2). The funnel plots did not indicate any bias and the $I^{2}$ values were mostly less than $40 \%$. However, for the analyses of TPO antibody-positive women, the $I^{2}$ values were $77 \%$ 

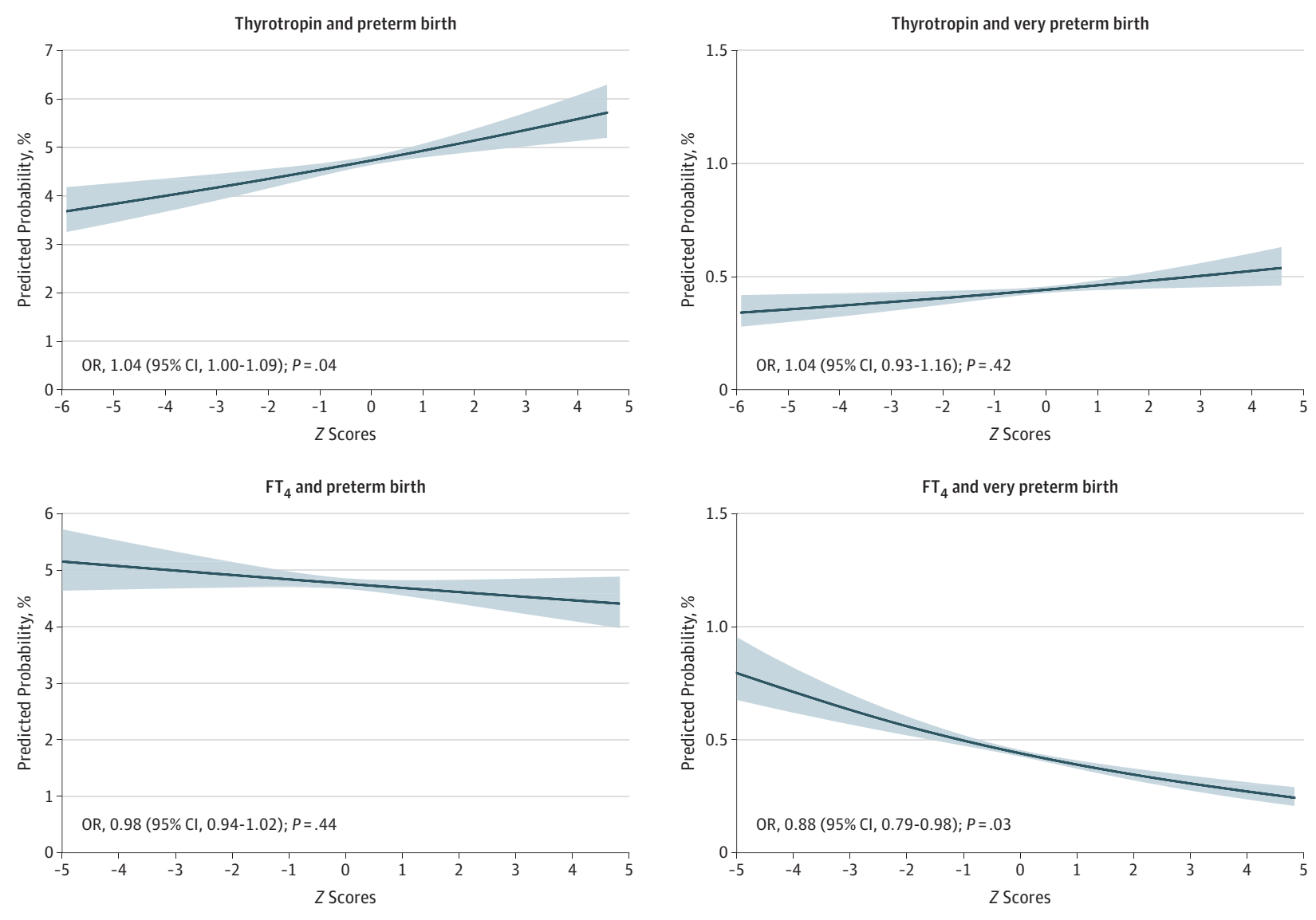

Preterm birth was defined as less than 37 weeks' gestational age and very preterm birth was defined as less than 32 weeks' gestational age. The thyrotropin and $\mathrm{FT}_{4}$ concentrations for all cohorts were log transformed and then standardized to population-specific standard deviation scores after

removal of outliers ( \pm 4 SD from the mean) to enable comparison between different cohorts and assays. All analyses were adjusted for maternal age, body mass index, ethnicity, smoking, parity, gestational age at blood sampling, and fetal sex. OR indicates odds ratio.

for preterm birth and 69\% for very preterm birth (eFigure 2 in Supplement 2).

\section{Discussion}

Subclinical hypothyroidism, isolated hypothyroxinemia, and TPO antibody positivity were associated with higher risk for preterm birth. Isolated hypothyroxinemia and TPO antibody positivity were associated with higher risk for very preterm birth. The association of TPO antibody positivity with preterm birth did not appear to be related to differences in thyroid function, but was modified by the thyrotropin concentration as exemplified by the higher risk of preterm birth in TPO antibody-positive women and a thyrotropin concentration above $4.0 \mathrm{mIU} / \mathrm{L}$.

Because randomized trials of treatment of thyroid function test abnormalities during pregnancy are scarce, observational studies form the basis of clinical guidelines on thyroid function and pregnancy. ${ }^{20}$ However, previous observational studies on the association of thyroid function and autoimmunity with preterm birth have used widely different defini- tions for thyroid function test abnormalities. This variation limits the ability to develop clearly defined recommendations in international guidelines and perform aggregate data meta-analysis. Furthermore, most studies lacked statistical power to study the risk of very preterm birth, a more clinically relevant outcome related to high mortality and morbidity, the demand for specialist neonatal services, and financial burdens. ${ }^{22-24}$ By collecting individual participant data, the current study allowed for standardization of the definition of thyroid function test abnormalities.

To our knowledge, the current study is the first individual participant data meta-analysis that shows isolated hypothyroxinemia is associated with a higher risk of preterm birth and very preterm birth and produced estimates in the same range as well-known risk factors such as adolescent or older maternal age, low BMI, obesity, smoking, and bacterial vaginosis. ${ }^{12,13,25-27}$ These results are in line with those from a previous population-based study, but opposite to those from an aggregate data meta-analysis including 5 studies. ${ }^{15,28}$ Isolated hypothyroxinemia is a pregnancy-specific disease entity with a multifactorial underlying pathophysiology that has remained relatively understudied. ${ }^{29}$ 


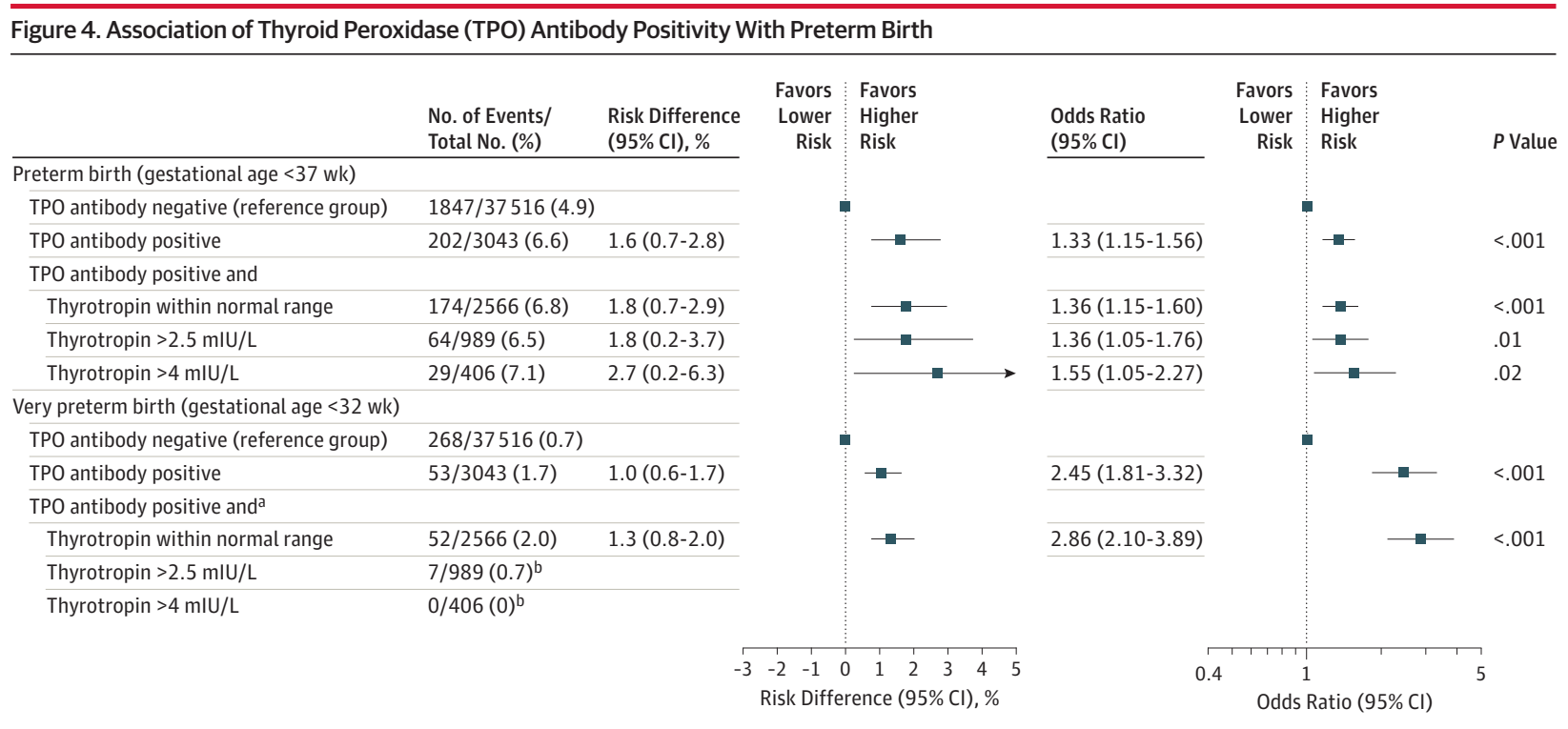

All analyses were adjusted for maternal age, body mass index, ethnicity, smoking, parity, gestational age at blood sampling, and fetal sex. Absolute differences and corresponding $95 \% \mathrm{Cls}$ were back-calculated from the results of multivariable models and adjusted for baseline risk imprecision.

a Thyroid peroxidase antibody-positive women and thyrotropin (often referred to as thyroid-stimulating hormone) concentrations within the normal range or higher than $2.5 \mathrm{mIU} / \mathrm{L}$ and $4 \mathrm{mIU} / \mathrm{L}$ were compared with TPO antibody-negative women regardless of their thyrotropin concentration.

${ }^{\mathrm{b}}$ There were too few samples to conduct a reliable analysis.
Available evidence predominantly links isolated hypothyroxinemia to suboptimal neurocognitive development of the offspring, ${ }^{29,30}$ but international guidelines do not recommend levothyroxine treatment in women with isolated hypothyroxinemia because randomized clinical trials have not proven a beneficial effect on child $\mathrm{IQ} .{ }^{2 \mathrm{O}}$ In 2 large randomized clinical trials in which women with isolated hypothyroxinemia were treated with levothyroxine during the first half of pregnancy, there was no statistically significant difference in preterm birth with treatment. In 1 trial of pregnant women with either subclinical hypothyroidism or isolated hypothyroxinemia, preterm birth occurred in $5.6 \%$ of the levothyroxine group vs $7.9 \%$ of the control group. ${ }^{31}$ In the second trial with pregnant women who had isolated hypothyroxinemia, preterm birth occurred in $12 \%$ of the levothyroxine group vs $8 \%$ of the placebo group and birth at less than 34 weeks' gestational age occurred in $4 \%$ of the levothyroxine group and $3 \%$ of the placebo group. ${ }^{32}$

Consistent with the results of the current study, women with isolated hypothyroxinemia in the 2 trials $^{31,32}$ had higher preterm birth rates than euthyroid women did in the current metaanalysis. The results of the current study provide further insights on potential risks of isolated hypothyroxinemia that may help to optimize clinical decision-making strategies, taking into account all potential harms and benefits of screening programs and subsequent levothyroxine treatment. Further studies will be required to elucidate the underlying pathophysiology of isolated hypothyroxinemia and to study if it is also a risk factor for other adverse pregnancy outcomes.

In the current study, TPO antibody positivity was associated with a higher risk of preterm birth, consistent with previous smaller meta-analyses using aggregate data and studies on miscarriage. ${ }^{12,13}$ Two recent clinical trials showed that preconception treatment of euthyroid TPO antibody-positive women who had either a previous miscarriage or fertility treatment with low-dose levothyroxine neither reduced the rate of preterm birth, nor any other pregnancy or neonatal outcomes. ${ }^{33,34}$ These studies did not include women with thyroid function test abnormalities. ${ }^{33,34}$ The results of the current study show a higher risk for TPO antibody-positive women and a thyrotropin concentration higher than $4.0 \mathrm{mIU} / \mathrm{L}$, which confirms the results from a small randomized clinical trial showing that levothyroxine treatment in TPO antibodypositive women lowers preterm birth rates only if the thyrotropin concentration was higher than $4.0 \mathrm{mIU} / \mathrm{L} .{ }^{35}$

Taken together, the results of this study are in alignment with the recommendation of the American Thyroid Association that different thyrotropin cutoffs should be used for TPO antibody-positive women vs TPO antibody-negative women. ${ }^{20}$ A sensitivity analysis showed that the association of subclinical hypothyroidism with preterm birth was no longer apparent after additional adjustment for TPO antibody positivity, suggesting that it is the TPO antibody positivity that occurs in one-third of women with subclinical hypothyroidism that underlies any associations of subclinical hypothyroidism with preterm birth. However, this study lacked statistical power for a subgroup analysis on the association of TPO antibody positivity in combination with a thyrotropin concentration higher than $2.5 \mathrm{mIU} / \mathrm{L}$ for preterm birth or higher than $4.0 \mathrm{mIU} / \mathrm{L}$ for very preterm birth.

There remains an important role for observational studies in the evaluation of thyroid function test abnormalities during pregnancy. For example, observational studies have indicated that the interpretation of the results of large randomized 
trials ${ }^{31-34}$ may be limited because of risks related to potential overtreatment, a late start of levothyroxine therapy, or sole inclusion of women with normal thyroid. ${ }^{36-39}$ Moreover, observational studies are needed to study detrimental clinical outcomes with a relatively low incidence such as very preterm birth, given the impracticality of randomized trials for such outcomes. For example, to study if levothyroxine therapy could reverse excess preterm birth risks as identified in this study for isolated hypothyroxinemia, TPO antibody positivity, and TPO antibody positivity with a thyrotropin concentration greater than $4.0 \mathrm{mIU} / \mathrm{L}$, a total of 3674,6090 , and 3814 women, respectively, would have to be randomized, which in the case of population screening would translate to the screening of 196 470, 95 530, and 448706 women (with a 2-sided a level of .05, a power level of $80 \%$, and a lost to follow-up rate or declined participation rate of $15 \%$ ).

The results of the current study do not change the consideration that there is currently insufficient evidence for a benefit of routine thyroid function screening in pregnant women; the potential harms and benefits of levothyroxine for other clinically meaningful outcomes should be taken into account as well. The results support the concept of a reflex TPO antibody measurement in women with a thyrotropin concentration higher than $4.0 \mathrm{mIU} / \mathrm{L}$, and gestational thyrotropin monitoring for TPO antibody-positive women prior to conception. The current study in itself does not validate a reflex $\mathrm{FT}_{4}$ concentration or TPO antibody measurement for women with a normal thyrotropin concentration until further randomized trials are performed.

The results of the current study add to the limited knowledge on the complicated and multifactorial mechanisms underlying preterm and very preterm birth. ${ }^{40}$ Because thyroid hormone regulates key processes in placental and fetal growth and development, the associations for $\mathrm{FT}_{4}$ concentration could be mediated via effects on either placental function, fetal growth, or both..$^{8,9,11,40,41}$ Alternatively, thyroid hormone and TPO antibody positivity could be involved in infectious and inflammatory pathways leading to preterm birth. ${ }^{42,43}$ Another potential mechanism by which low thyroid hormone availability could be associated with preterm birth is that it may lead to an earlier onset of labor via an increase in oxytocin and vasopressin, but a decrease in progesterone, or through thyroid hormone effects specific for the cervix, endometrium, or fe- tal membranes. ${ }^{7,44-47}$ Further studies on pathways via which thyroid hormone is involved in the pathogenesis of preterm birth are needed to further optimize risk identification.

\section{Limitations}

This study has several limitations. First, there was a lack of statistical power to optimally investigate the risk of very preterm birth in specific clinically relevant subgroups. Although very preterm birth is clinically more relevant than late preterm birth, the relatively sparse occurrence of TPO antibody positivity combined with a thyrotropin concentration higher than 2.5 or $4.0 \mathrm{mIU} / \mathrm{L}$ necessitates an even larger data set than that available in the current study to examine associations with very preterm birth.

Second, only 5 of the 19 studies had data available on thyroglobulin antibodies. Although this association was studied in a large number of women, data were only available for $40.0 \%$ of included women, which may have affected the results.

Third, pregnancy-related changes in thyroid-binding proteins could interfere with $\mathrm{FT}_{4}$ immunoassays. However, the use of assay-specific reference ranges as advocated by the current American Thyroid Association guidelines and standardization of $\mathrm{FT}_{4}$ concentrations per cohort or assay should mostly eliminate the potential between-assay differences in absolute $\mathrm{FT}_{4}$ concentrations.

Fourth, studies that were published while conducting statistical analyses for the current study could not be included. Fifth, because the included studies were observational, residual or unmeasured confounding cannot be excluded. However, the analysis of individual participant data allowed for adjustment for multiple relevant confounders. Sixth, causality cannot be determined from observational studies.

\section{Conclusions}

Among pregnant women without overt thyroid disease, subclinical hypothyroidism, isolated hypothyroxinemia, and TPO antibody positivity were significantly associated with higher risk of preterm birth. These results provide insights toward optimizing clinical decision-making strategies that should consider the potential harms and benefits of screening programs and levothyroxine treatment during pregnancy.

\section{ARTICLE INFORMATION}

Accepted for Publication: July 8, 2019.

Authors/The Consortium on Thyroid and Pregnancy-Study Group on Preterm Birth: Tim I. M. Korevaar, MD, PhD; Arash Derakhshan, MD, MSc; Peter N. Taylor, MRCP, PhD; Marcel Meima, PhD; Liangmiao Chen, MD, PhD; Sofie Bliddal, MD; David M. Carty, MD; Margreet Meems, MD, PhD; Bijay Vaidya, FRCP, PhD; Beverley Shields, PhD; Farkhanda Ghafoor, PhD; Polina V. Popova, MD, PhD; Lorena Mosso, MD, PhD; Emily Oken, MD, MPH; Eila Suvanto, MD, PhD; Aya Hisada, PhD; Jun Yoshinaga, PhD; Suzanne J. Brown; Judith Bassols, MD; Juha Auvinen, MD, PhD; Wichor M. Bramer; Abel López-Bermejo, MD, PhD; Colin Dayan, MD, PhD; Laura Boucai, MD, MSc; Marina
Vafeiadi, PhD; Elena N. Grineva, MD, PhD; Alexandra S. Tkachuck, MD; Victor J. M. Pop, MD, PhD; Tanja G. Vrijkotte, PhD; Mònica Guxens, MD, PhD; Leda Chatzi, MD, PhD; Jordi Sunyer, MD, PhD; Ana Jiménez-Zabala, PhD; Isolina Riaño, MD, PhD; Mario Murcia, PhD; Xuemian Lu, MD, PhD; Shafqat Mukhtar, MD; Christian Delles, MD; Ulla Feldt-Rasmussen, MD, DMSc; Scott M. Nelson, MRCOG, PhD; Erik K. Alexander, MD; Layal Chaker, MD, PhD; Tuija Männistö, MD, PhD; John P. Walsh, MB, PhD; Elizabeth N. Pearce, MD; Eric A. P. Steegers, MD, PhD; Robin P. Peeters, MD, PhD.

Affiliations of Authors/The Consortium on Thyroid and Pregnancy-Study Group on Preterm Birth: Department of Internal Medicine, Erasmus University Medical Center, Rotterdam, the Netherlands (Korevaar, Derakhshan, Meima, Chaker, Peeters); Academic Center for Thyroid Diseases, Erasmus University Medical Center, Rotterdam, the Netherlands (Korevaar, Derakhshan, Meima, Chaker, Peeters); Thyroid Research Group, Systems Immunity Research Institute, School of Medicine, Cardiff University, Cardiff, England (Taylor); Department of Endocrinology and Rui'an Center of the Chinese-American Research Institute for Diabetic Complications, Third Affiliated Hospital of Wenzhou Medical University, Wenzhou, China (Chen, Lu): Department of Medical Endocrinology, Copenhagen University Hospital, Rigshospitalet, Copenhagen, Denmark (Bliddal, Feldt-Rasmussen); Department of Diabetes, Endocrinology, and Clinical Pharmacology, Glasgow Royal Infirmary, 
Glasgow, Scotland (Carty); Institute of Cardiovascular and Medical Sciences, University of Glasgow, Glasgow, Scotland (Carty, Delles); Departments of Medical and Clinical Psychology, Tilburg University, Tilburg, the Netherlands (Meems, Pop); Department of Endocrinology, Royal Devon and Exeter Hospital NHS Foundation Trust, University of Exeter Medical School, Exeter, England (Vaidya); Institute of Biomedical and Clinical Science, University of Exeter Medical School, Exeter, England (Shields); National Health Research Complex, Shaikh Zayed Medical Complex Lahore, Pakistan (Ghafoor); Almazov National Medical Research Centre, St Petersburg, Russia (Popova, Grineva, Tkachuck); Department of Internal Diseases and Endocrinology, St Petersburg Pavlov State Medical University, St Petersburg, Russia (Popova, Grineva, Tkachuck); Department of Endocrinology, Pontificia Universidad Catolica de Chile, Santiago (Mosso); Division of Chronic Disease Research Across the Lifecourse, Department of Population Medicine, Harvard Medical School, Boston, Massachusetts (Oken); Harvard Pilgrim Health Care Institute, Boston, Massachusetts (Oken); Department of Nutrition, Harvard T. H. Chan School of Public Health, Boston, Massachusetts (Oken); Department of Obstetrics and Gynecology and Medical Research Center Oulu, University of Oulu, Oulu, Finland (Suvanto); Center for Preventive Medical Science, Chiba University, Chiba, Japan (Hisada); Faculty of Life Sciences, Toyo University, Gunma, Japan (Yoshinaga); Department of Endocrinology and Diabetes, Sir Charles Gairdner Hospital, Nedlands, Australia (Brown);

Maternal-Fetal Metabolic Research Group, Girona Biomedical Research Institute, Dr Josep Trueta Hospital, Girona, Spain (Bassols, Walsh); Medical Research Center Oulu, Oulu University Hospital, Center for Life Course Health Research, University of Oulu, Oulu, Finland (Auvinen); Medical Library, Erasmus University Medical Center, Rotterdam, the Netherlands (Bramer); Pediatric Endocrinology Research Group, Girona Biomedical Research Institute, Dr Josep Trueta Hospital, Girona, Spain (López-Bermejo); Thyroid Research Group, Institute of Molecular and Experimental Medicine School of Medicine, Cardiff University, Cardiff, England (Dayan); Division of Endocrinology, Department of Medicine, Memorial Sloan-Kettering Cancer Center, Weill Cornell University, New York, New York (Boucai); Department of Social Medicine, University of Crete, Heraklion, Greece (Vafeiadi); Department of Public Health, Amsterdam University Medical Center, University of Amsterdam, Amsterdam Public Health Research Institute, Amsterdam, the Netherlands (Vrijkotte); ISGlobal, Centre for Research in Environmental Epidemiology, Barcelona, Spain (Guxens, Sunyer); Pompeu Fabra University, Barcelona, Spain (Guxens, Sunyer); Spanish Consortium for Research on Epidemiology and Public Health, Instituto de Salud Carlos III, Madrid, Spain (Guxens, Sunyer, Riaño, Murcia); Department of Child and Adolescent Psychiatry/Psychology, Erasmus University Medical Center, Sophia Children's Hospital, Rotterdam, the Netherlands (Guxens); Keck School of Medicine, Department of Preventive Medicine, University of Southern California, Los Angeles (Chatzi); Hospital del Mar Medical Research Institute, Barcelona, Spain (Sunyer); Biodonostia Health Research Institute, San Sebastian, Spain (Jiménez-Zabala); Public Health Division of Gipuzkoa, Basque Government, San Sebastian,
Spain (Jiménez-Zabala); AGC Pediatrics, Hospita Universitario Central de Asturias, Oviedo, Spain (Riaño); Epidemiology and Environmental Health Joint Research Unit, FISABIO-Universitat Jaume I-Universitat de València, Valencia, Spain (Murcia) Department of Gynecology and Obstetrics, Shaikh Zayed Medical Complex, Lahore, Pakistan (Mukhtar); School of Medicine, University of Glasgow, Glasgow, Scotland (Nelson); National Institute for Health Research, Bristol Biomedical Research Centre, Bristol, England (Nelson); Division of Endocrinology, Hypertension, and Diabetes, Brigham and Women's Hospital, Harvard Medical School, Boston, Massachusetts (Alexander); Northern Finland Laboratory Center Nordlab and Medical Research Center Oulu, Oulu University Hospital and University of Oulu, Oulu, Finland (Männistö); Medical School, University of Western Australia, Crawley (Walsh); Section of Endocrinology, Diabetes, and Nutrition, Boston University School of Medicine, Boston, Massachusetts (Pearce); Department of Obstetrics and Gynaecology, Erasmus University Medical Center, Rotterdam, the Netherlands (Steegers).

Author Contributions: Drs Korevaar and Derakhshan had full access to all of the data in the study and take responsibility for the integrity of the data and the accuracy of the data analysis. Drs Korevaar and Derakhshan contributed equally. Concept and design: Korevaar, Derakhshan, Bliddal Ghafoor, Oken, Suvanto, Delles, Feldt-Rasmussen, Alexander, Nelson, Chaker, Männistö, Peeters. Acquisition, analysis, or interpretation of data: Korevaar, Derakhshan, Taylor, Meima, Chen, Bliddal, Carty, Meems, Vaidya, Shields, Popova, Mosso Oken, Hisada, Yoshinaga, Brown, Bassols, Auvinen Bramer, López-Bermejo, Dayan, Boucai, Vafeiadi, Grineva, Tkachuck, Pop, Vrijkotte, Guxens, Chatzi, Sunyer, Jiménez-Zabala, Riaño, Murcia, Lu, Mukhtar, Delles, Alexander, Nelson, Chaker, Männistö, Walsh, Pearce, Steegers, Peeters. Drafting of the manuscript: Korevaar, Derakhshan, Meima, Mosso, Suvanto, Tkachuck, Alexander, Peeters.

Critical revision of the manuscript for important intellectual content: Korevaar, Derakhshan, Taylor, Meima, Chen, Bliddal, Carty, Meems, Vaidya, Shields, Ghafoor, Popova, Oken, Hisada, Yoshinaga, Brown, Bassols, Auvinen, Bramer, López-Bermejo Dayan, Boucai, Vafeiadi, Grineva, Pop, Vrijkotte, Guxens, Chatzi, Sunyer, Jiménez-Zabala, Riaño, Murcia, Lu, Mukhtar, Delles, Feldt-Rasmussen, Alexander, Nelson, Chaker, Männistö, Walsh, Pearce, Steegers, Peeters.

Statistical analysis: Korevaar, Derakhshan, Brown, Pop, Chaker, Walsh, Peeters.

Obtained funding: Korevaar, Bliddal, Oken, Vrijkotte, Guxens, Chatzi, Delles, Nelson, Walsh, Peeters.

Administrative, technical, or material support: Korevaar, Derakhshan, Bliddal, Meems, Ghafoor, Popova, Mosso, Oken, Hisada, Yoshinaga, Brown, Bassols, Auvinen, Dayan, Vafeiadi, Grineva, Tkachuck, Jiménez-Zabala, Riaño, Lu, Mukhtar, Peeters.

Supervision: Korevaar, Meima, Chen, Bliddal, Mosso, Boucai, Chatzi, Sunyer, Riaño, Alexander, Steegers, Peeters.

Conflict of Interest Disclosures: Dr Korevaar reported receiving personal fees from Berlin Chemie, Goodlife Healthcare, and Quidel. Dr Vaidya reported receiving honoraria from Berlin-Chime. Dr Oken reported receiving grants from the
National Institutes of Health. Dr Vrijkotte reported receiving grants from the Netherlands Organization for Health Research and Development. Dr Delles reported receiving grants from the Chief Scientist Office (Scotland) and the British Heart Foundation. Dr Nelson reported receiving grants from the Chief Scientist Office (Scotland). Dr Chaker reported serving as a consultant to Pfizer. Dr Männistö reported serving as a consultant to Abbott Diagnostics and Roche. Dr Pearce reported receiving grants from the Sociedad Quimica y Minera de Chile and personal fees from the Institut Biochimique SA. Dr Peeters reported serving as a consultant to Berlin-Chemie AG, GoodLife Fertility $B V$, and Institut Biochimique SA. No other disclosures were reported.

Funding/Support: This work was supported by replication studies grant 401.16 .020 from the Netherlands Organization for Scientific Research. Cohort-specific grants appear in Supplement 2.

Role of the Funder/Sponsor: The Netherlands Organization for Scientific Research had no role in the design and conduct of the study; collection, management, analysis, and interpretation of the data; preparation, review, or approval of the manuscript; and decision to submit the manuscript for publication.

Additional Contributions: We gratefully acknowledge the contributions of the study participants across all cohorts included in the current study and extend our gratitude to study staff, data managers, general practitioners, hospitals, and midwives who have made these separate studies possible. We also gratefully acknowledge the contributions of Amna Pirzada, MD (Shifa College of Medicine, Shifa Tameer-e-Millat University), for data management; and Andrew Hattersley, FRS, Beatrice Knight, MD, Rachel Freathy, PhD, and Robin Beaumont, PhD (all with the University of Exeter), for data collection and management. None of these persons received compensation for their contributions.

\section{REFERENCES}

1. Liu L, Oza S, Hogan D, et al. Global, regional, and national causes of under-5 mortality in 2000-15: an updated systematic analysis with implications for the sustainable development goals. Lancet. 2016; 388(10063):3027-3035. doi:10.1016/S0140-6736 (16)31593-8

2. Nosarti C, Reichenberg A, Murray RM, et al. Preterm birth and psychiatric disorders in young adult life. Arch Gen Psychiatry. 2012;69(6):E1-E8. doi:10.1001/archgenpsychiatry.2011.1374

3. Vikse BE, Irgens LM, Leivestad $T$, Hallan $S$, Iversen BM. Low birth weight increases risk for end-stage renal disease. J Am Soc Nephrol. 2008;19 (1):151-157. doi:10.1681/ASN.2007020252

4. Blencowe $\mathrm{H}$, Cousens $\mathrm{S}$, Oestergaard MZ, et al. National, regional, and worldwide estimates of preterm birth rates in the year 2010 with time trends since 1990 for selected countries: a systematic analysis and implications. Lancet. 2012;379(9832):2162-2172. doi:10.1016/S0140-6736 (12)60820-4

5. Menon R. Spontaneous preterm birth, a clinical dilemma: etiologic, pathophysiologic and genetic heterogeneities and racial disparity. Acta Obstet Gynecol Scand. 2008;87(6):590-600. doi:10.1080/ 00016340802005126 
6. Ramadurai SM, Nielsen HC, Chen Y, Hatzis D, Sosenko IR. Differential effects in vivo of thyroid hormone on the expression of surfactant phospholipid, surfactant protein mRNA and antioxidant enzyme mRNA in fetal rat lung. Exp Lung Res. 1998;24(5):641-657. doi:10.3109/ 01902149809099585

7. Ciosek J, Drobnik J. Vasopressin and oxytocin release and the thyroid function. $J$ Physiol Pharmacol. 2004;55(2):423-441.

8. Chen $\mathrm{CY}$, Chen $\mathrm{CP}$, Lin KH. Biological functions of thyroid hormone in placenta. Int J Mol Sci. 2015;16 (2):4161-4179. doi:10.3390/ijms16024161

9. Barjaktarovic M, Korevaar TI, Chaker L, et al. The association of maternal thyroid function with placental hemodynamics. Hum Reprod. 2017;32(3): 653-661.

10. Dong AC, Stagnaro-Green A. Differences in diagnostic criteria mask the true prevalence of thyroid disease in pregnancy: a systematic review and meta-analysis. Thyroid. 2019;29(2):278-289. doi:10.1089/thy.2018.0475

11. Korevaar TIM, Medici M, Visser TJ, Peeters RP. Thyroid disease in pregnancy: new insights in diagnosis and clinical management. Nat Rev Endocrinol. 2017;13(10):610-622. doi:10.1038/nrendo. 2017.93

12. Thangaratinam S, Tan A, Knox E, Kilby MD, Franklyn J, Coomarasamy A. Association between thyroid autoantibodies and miscarriage and preterm birth: meta-analysis of evidence. BMJ. 2011;342:d2616. doi:10.1136/bmj.d2616

13. He X, Wang P, Wang Z, He X, Xu D, Wang B. Thyroid antibodies and risk of preterm delivery: a meta-analysis of prospective cohort studies. Eur J Endocrinol. 2012;167(4):455-464. doi:10.1530/EJE12-0379

14. Maraka S, Ospina NM, O'Keeffe DT, et al. Subclinical hypothyroidism in pregnancy: a systematic review and meta-analysis. Thyroid. 2016;26(4):580-590. doi:10.1089/thy.2015.0418

15. Sheehan PM, Nankervis $A$, Araujo Júnior $E$, Da Silva Costa F. Maternal thyroid disease and preterm birth: systematic review and meta-analysis. J Clin Endocrinol Metab. 2015;100 (11):4325-4331. doi:10.1210/jc.2015-3074 16. Korevaar TI, Taylor PN, Dayan CM, Peeters RP. An invitation to join the consortium on thyroid and pregnancy. Eur Thyroid J. 2016;5(4):277. doi:10. 1159/000452331

17. Korevaar TI, Taylor PN, Dayan CM, Peeters RP. An invitation to join the consortium on thyroid and pregnancy. Obstet Gynecol. 2016;128(4):913. doi:10. 1097/AOG.0000000000001670

18. Jolani S, Debray TP, Koffijberg H, van Buuren $S$, Moons KG. Imputation of systematically missing predictors in an individual participant data meta-analysis: a generalized approach using MICE. Stat Med. 2015;34(11):1841-1863. doi:10.1002/sim. 6451

19. van Buuren S, Groothuis-Oudshoorn K. MICE: multivariate imputation by chained equations in $\mathrm{R}$. J Stat Softw. 2011;1(3):26.

20. Alexander EK, Pearce EN, Brent GA, et al. 2017 guidelines of the American Thyroid Association for the diagnosis and management of thyroid disease during pregnancy and the postpartum. Thyroid. 2017;27(3):315-389. doi:10.1089/thy.2016.0457
21. Korevaar TIM, Pop VJ, Chaker L, et al. Dose dependency and a functional cutoff for TPO-antibody positivity during pregnancy. J Clin Endocrinol Metab. 2018;103(2):778-789. doi:10. 1210/jc.2017-01560

22. Boyle EM, Johnson S, Manktelow B, et al. Neonatal outcomes and delivery of care for infants born late preterm or moderately preterm: a prospective population-based study. Arch Dis Child Fetal Neonatal Ed. 2015;100(6):F479-F485. doi:10.1136/archdischild-2014-307347

23. Vohr B. Long-term outcomes of moderately preterm, late preterm, and early term infants. Clin Perinatol. 2013;40(4):739-751. doi:10.1016/j.clp. 2013.07.006

24. Boyle EM, Poulsen G, Field DJ, et al. Effects of gestational age at birth on health outcomes at 3 and 5 years of age: population based cohort study. BMJ. 2012;344:e896. doi:10.1136/bmj.e896

25. Bhattacharya S, Raja EA, Mirazo ER, et al. Inherited predisposition to spontaneous preterm delivery. Obstet Gynecol. 2010;115(6):1125-1133. doi: 10.1097/AOG.Ob013e3181dffcdb

26. Leitich $H$, Bodner-Adler $B$, Brunbauer $M$, Kaider A, Egarter C, Husslein P. Bacterial vaginosis as a risk factor for preterm delivery: a meta-analysis. Am J Obstet Gynecol. 2003;189(1):139-147. doi:10.1067/ mob.2003.339

27. Shah NR, Bracken MB. A systematic review and meta-analysis of prospective studies on the association between maternal cigarette smoking and preterm delivery. Am J Obstet Gynecol. 2000 182(2):465-472. doi:10.1016/S0002-9378(00) 70240-7

28. Korevaar TIM, Steegers EAP, Chaker L, et al. Thyroid function and premature delivery in TPO antibody-negative women: the added value of hCG. J Clin Endocrinol Metab. 2017;102(9):3360-3367. doi:10.1210/jc.2017-00846

29. Dosiou C, Medici M. Management of endocrine disease: isolated maternal hypothyroxinemia during pregnancy: knowns and unknowns. Eur J Endocrinol. 2017;176(1):R21-R38. doi:10.1530/EJE-16-0354

30. Korevaar TIM, Tiemeier H, Peeters RP. Clinical associations of maternal thyroid function with foetal brain development: epidemiological interpretation and overview of available evidence. Clin Endocrinol (Oxf). 2018;89(2):129-138. doi:10. 1111/cen.13724

31. Lazarus JH, Bestwick JP, Channon S, et al. Antenatal thyroid screening and childhood Cognitive function. N Engl J Med. 2012;366(6):493501. doi:10.1056/NEJMoa1106104

32. Casey BM, Thom EA, Peaceman AM, et al; Eunice Kennedy Shriver National Institute of Child Health and Human Development Maternal-Fetal Medicine Units Network. Treatment of subclinical hypothyroidism or hypothyroxinemia in pregnancy. N Engl J Med. 2017;376(9):815-825. doi:10.1056/ NEJMoa1606205

33. Dhillon-Smith RK, Middleton LJ, Sunner KK, et al. Levothyroxine in women with thyroid peroxidase antibodies before conception. NEngl J Med. 2019;380(14):1316-1325. doi:10.1056/ NEJMoa1812537

34. Wang $\mathrm{H}$, Gao H, Chi $\mathrm{H}$, et al. Effect of levothyroxine on miscarriage among women with normal thyroid function and thyroid autoimmunity undergoing in vitro fertilization and embryo transfer: a randomized clinical trial. JAMA. 2017;318 (22):2190-2198. doi:10.1001/jama.2017.18249

35. Nazarpour S, Ramezani Tehrani F, Simbar M, Tohidi M, Alavi Majd H, Azizi F. Effects of levothyroxine treatment on pregnancy outcomes in pregnant women with autoimmune thyroid disease. Eur J Endocrinol. 2017;176(2):253-265. doi: 10.1530/EJE-16-0548

36. Jansen TA, Korevaar TIM, Mulder TA, et al. Maternal thyroid function during pregnancy and child brain morphology: a time window-specific analysis of a prospective cohort [published online June 28, 2019]. Lancet Diabetes Endocrinol. doi:10. 1016/S2213-8587(19)30153-6

37. Maraka S, Mwangi R, McCoy RG, et al. Thyroid hormone treatment among pregnant women with subclinical hypothyroidism: US national assessment. BMJ. 2017;356:i6865. doi:10.1136/bmj. i6865

38. Korevaar TI, Muetzel R, Medici M, et al. Association of maternal thyroid function during early pregnancy with offspring IQ and brain morphology in childhood: a population-based prospective cohort study. Lancet Diabetes Endocrinol. 2016;4(1):35-43. doi:10.1016/S22138587(15)00327-7

39. Korevaar TIM, Chaker L, Peeters RP. Improving the clinical impact of randomised trials in thyroidology. Lancet Diabetes Endocrinol. 2018;6 (7):523-525. doi:10.1016/S2213-8587(17)30316-9

40. Romero R, Dey SK, Fisher SJ. Preterm labor: one syndrome, many causes. Science. 2014;345 (6198):760-765. doi:10.1126/science.1251816

41. Goldenberg RL, Culhane JF, lams JD, Romero R. Epidemiology and causes of preterm birth. Lancet. 2008;371(9606):75-84. doi:10.1016/S0140-6736 (08)60074-4

42. Goldenberg RL, Hauth JC, Andrews WW. Intrauterine infection and preterm delivery. N Engl J Med. 2000;342(20):1500-1507. doi:10.1056/ NEJM200005183422007

43. De Vito P, Incerpi S, Pedersen JZ, Luly P, Davis FB, Davis PJ. Thyroid hormones as modulators of immune activities at the cellular level. Thyroid. 2011; 21(8):879-890. doi:10.1089/thy.2010.0429

44. Datta M, Roy P, Banerjee J, Bhattacharya S Thyroid hormone stimulates progesterone release from human luteal cells by generating a proteinaceous factor. J Endocrinol. 1998;158(3):319325. doi:10.1677/joe.0.1580319

45. Maruo T, Matsuo $H$, Mochizuki M. Thyroid hormone as a biological amplifier of differentiated trophoblast function in early pregnancy. Acta Endocrinol (Copenh). 1991;125(1):58-66. doi:10. 1530/acta.0.1250058

46. Akerlund $M$. Vasopressin and oxytocin in normal reproduction and in the pathophysiology of preterm labour and primary dysmenorrhea: development of receptor antagonists for therapeutic use in these conditions. Rocz Akad Med Bialymst. 2004:49:18-21.

47. Thornton S, Baldwin PJ, Harris PA, et al. The role of arginine vasopressin in human labour: functional studies, fetal production and localisation of V1a receptor mRNA. BJOG. 2002;109(1):57-62. doi:10.1111/j.1471-0528.2002.01132.x 